\title{
2D vs 3D gamma analysis: Establishment of comparable clinical action limits
}

\author{
Kiley B Pulliam ${ }^{1}$, Jessie Y Huang ${ }^{1}$, Ryan Bosca ${ }^{1}$, David Followill ${ }^{2}$, Stephen F Kry ${ }^{2}$ \\ ${ }^{1}$ The University of Texas Graduate School of Biomedical Sciences at Houston, Houston, TX, USA. \\ ${ }^{2}$ Department of Radiation Physics, The University of Texas MD Anderson Cancer Center, Houston, TX, USA.
}

Received March 19, 2014; Published Online April 08, 2014

[Presented at the Young Investigator's Symposium at the 2014 Annual Meeting of

Southwest Chapter of American Association of Physicists in Medicine (AAPM) in San Antonio, Texas, USA]

\section{Conference Proceeding}

\begin{abstract}
Purpose: As clinics begin to use 3D metrics for intensity-modulated radiation therapy (IMRT) quality assurance; these metrics will often produce results different from those produced by their $2 \mathrm{D}$ counterparts. $3 \mathrm{D}$ and $2 \mathrm{D}$ gamma analyses would be expected to produce different values, because of the different search space available. We compared the results of $2 \mathrm{D}$ and $3 \mathrm{D}$ gamma analysis (where both datasets were generated the same way) for clinical treatment plans.
\end{abstract}

Methods: 50 IMRT plans were selected from our database and recalculated using Monte Carlo. Treatment planning system-calculated ("evaluated") and Monte Carlo-recalculated ("reference") dose distributions were compared using 2D and 3D gamma analysis. This analysis was performed using a variety of dose-difference (5\%, 3\%, $2 \%$, and $1 \%$ ) and distance-to-agreement $(5,3,2$, and $1 \mathrm{~mm})$ acceptance criteria, low-dose thresholds $(5 \%, 10 \%$, and $15 \%$ of the prescription dose), and data grid sizes (1.0, 1.5, and $3.0 \mathrm{~mm})$. Each comparison was evaluated to determine the average $2 \mathrm{D}$ and $3 \mathrm{D}$ gamma and percentage of pixels passing gamma.

Results: Average gamma and percentage of passing pixels for each acceptance criterion demonstrated better agreement for $3 \mathrm{D}$ than for 2D analysis for every plan comparison. Average difference in the percentage of passing pixels between the 2D and $3 \mathrm{D}$ analyses with no low-dose threshold ranged from $0.9 \%$ to $2.1 \%$. Similarly, using a low-dose threshold resulted

Presenting author: Kiley B Pulliam; The University of Texas Graduate School of Biomedical Sciences at Houston, Houston, TX, USA.

Cite this article as:

Pulliam KB, Huang JY, Bosca R, Followill D, Kry SF. 2D vs. 3D gamma analysis: Establishment of comparable clinical action limits. Int J Cancer Ther Oncol 2014; 2(2):020231.

DOI: $10.14319 /$ ijcto.0202.31 in a differences ranging from $0.8 \%$ to $1.5 \%$. No appreciable differences in gamma with changes in the data density (constant difference: $0.8 \%$ for $2 \mathrm{D}$ vs. $3 \mathrm{D}$ ) were observed.

Conclusion: We found that 3D gamma analysis resulted in up to $2.9 \%$ more pixels passing than $2 \mathrm{D}$ analysis. Factors such as inherent dosimeter differences may be an important additional consideration to the extra dimension of available data that was evaluated in this study.
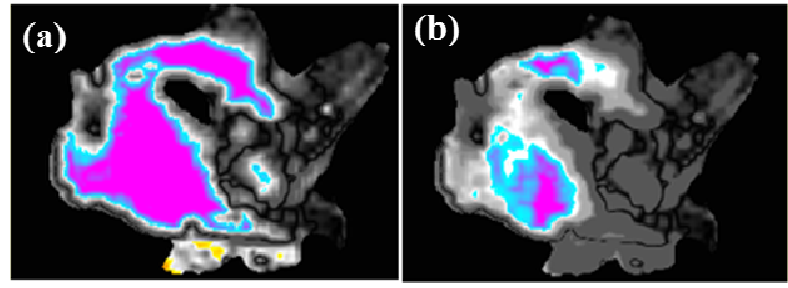

FIG. 1: (a) 2D and (b) 3D gamma maps of the same transverse slice of the gamma comparison in a homogenous QA phantom showing gamma failure (i.e. $\gamma>1$ ) in the 2D map (average $\gamma=1.04$ ) but passing in the 3D map (average $\gamma=0.52$ ) for the $3 \% / 3-\mathrm{mm}$ acceptance criteria with a $15 \%$ low-dose threshold.

Key Results:

2D vs. 3D gamma for different $Q A$ evaluation criteria: The average gamma value and percentage of pixels passing gamma for the comparisons of $2 \mathrm{D}$ and $3 \mathrm{D}$ gamma with a variety of dose-difference and DTA criteria for the 50 QA plans and 50 corresponding patient plans are shown in Table 1. Overall, for each acceptance criterion, the 3D gamma was lower than the 2D gamma (i.e., better agreement), on average for both the QA and patient plans. The differences between the average $2 \mathrm{D}$ and $3 \mathrm{D}$ gamma and percentage of pixels were statistically significant $(P<0.001)$ for both QA and patient plans. 
TABLE 1: Average 2D vs. 3D gammas and percentages of pixels passing gamma values for the (a) $50 \mathrm{QA}$ and (b) 50 patient plans at the $5 \% / 5-\mathrm{mm}$, $3 \% / 3-\mathrm{mm}, 2 \% / 2-\mathrm{mm}$, and $1 \% / 1-\mathrm{mm}$ acceptance criteria with no low-dose threshold and a 1-mm data grid.

\begin{tabular}{ccccc}
\hline \hline $\begin{array}{c}\text { Acceptance } \\
\text { criteria }\end{array}$ & Average 2D gamma & Average 3D gamma & $\begin{array}{c}\text { Average 2D percentage of } \\
\text { pixels passing gamma }\end{array}$ & $\begin{array}{c}\text { Average 3D percentage of } \\
\text { pixels passing gamma }\end{array}$ \\
\hline $5 \% / 5 \mathrm{~mm}$ & 0.22 & 0.20 & 98.3 & 98.7 \\
$3 \% / 3 \mathrm{~mm}$ & 0.37 & 0.33 & 96.6 & 97.4 \\
$2 \% / 2 \mathrm{~mm}$ & 0.56 & 0.51 & 93.2 & 94.9 \\
$1 \% / 1 \mathrm{~mm}$ & 1.18 & 1.10 & 79.1 & 80.9 \\
\hline \hline
\end{tabular}

(a)

\begin{tabular}{ccccc}
\hline \hline $\begin{array}{c}\text { Acceptance } \\
\text { criteria }\end{array}$ & Average 2D gamma & Average 3D gamma & $\begin{array}{c}\text { Average 2D percentage of } \\
\text { pixels passing gamma }\end{array}$ & $\begin{array}{c}\text { Average 3D percentage of } \\
\text { pixels passing gamma }\end{array}$ \\
\hline $5 \% / 5 \mathrm{~mm}$ & 0.25 & 0.22 & 98.0 & 98.9 \\
$3 \% / 3 \mathrm{~mm}$ & 0.44 & 0.37 & 94.2 & 96.4 \\
$2 \% / 2 \mathrm{~mm}$ & 0.71 & 0.59 & 88.8 & 91.7 \\
$1 \% / 1 \mathrm{~mm}$ & 1.85 & 1.58 & 74.9 & 77.0 \\
\hline \hline
\end{tabular}

(b)

Application to IMRT QA: In general, there are many differences between planar and volumetric analysis for IMRT QA. Individual planes in 2D analysis may miss problems that would be identified with $3 \mathrm{D}$ analysis, but could also highlight local regions where problems exist. Figure 1 shows a clinical plan in which the same single transverse slice failed 2D gamma QA $(\gamma=1.04)$ but easily passed 3D gamma QA $(\gamma=0.52)$. This sort of case likely represents a scenario where a plan "failed" based on $2 \mathrm{D}$ analysis that should have, in reality, passed, as indicated in the $3 \mathrm{D}$ analysis. 